\title{
Economic Evaluation of Tobramycin Inhalation Powder for the Treatment of Chronic Pulmonary Pseudomonas aeruginosa Infection in Patients with Cystic Fibrosis
}

\author{
Srilekha Panguluri ${ }^{1} \cdot$ Praveen Gunda $^{1} \cdot$ Laurie Debonnett $^{2} \cdot$ Kamal Hamed $^{2}$ (I)
}

Published online: 22 June 2017

(C) The Author(s) 2017. This article is an open access publication

\begin{abstract}
Background Chronic lung infection with Pseudomonas aeruginosa occurs in approximately $50 \%$ of patients with cystic fibrosis (CF). This infection further compromises lung function, and significantly contributes to the increased healthcare costs.

Objectives Inhaled tobramycin, used to manage $P$. aeruginosa infection in CF patients, is available as powder (tobramycin inhalation powder, TIP) and solution (tobramycin inhalation solution, TIS). Evidence suggests increased adherence with the use of TIP over TIS. Hence, this analysis aimed to evaluate the potential pharmacoeconomic benefit of increased adherence with TIP over TIS in the US setting.

Methods A patient-level simulation model was developed to compare TIP with TIS. Both costs and benefits were predicted over a 10-year time horizon from a payer's perspective, and were discounted annually at $3 \%$. All costs were presented in 2016 US dollars.

Results TIP was associated with greater quality-adjusted life-years (by 0.27 ) and lower total costs (by US $\$ 36,168$ ) as compared with TIS over a 10-year time horizon. TIPtreated patients experienced a decreased mean number of exacerbations than TIS-treated patients (39.24 vs 50.20). Furthermore, administration of TIP via the T-326 Inhaler was associated with significant cost savings per patient, because of the nebulizer required for administering TIS (by US\$1596) and exacerbation costs (by US\$76,531).
\end{abstract}

Kamal Hamed

kamal.hamed@novartis.com; kamalhamed1@aol.com

Novartis Healthcare Pvt. Ltd., Hyderabad, India

2 Novartis Pharmaceuticals Corporation, One Health Plaza, East Hanover, NJ 07936, USA
Probabilistic sensitivity analysis showed that TIP was dominant over TIS in $100 \%$ of the simulations.

Conclusion TIP is likely to be a more cost-effective treatment than TIS, and therefore may reduce the economic burden of $\mathrm{CF}$.

\section{Key Points}

This is the first cost-effectiveness analysis of tobramycin inhalation powder (TIP) versus tobramycin inhalation solution (TIS) for the treatment of chronic pulmonary Pseudomonas aeruginosa infection in patients with cystic fibrosis (CF) in the US setting, thus exploring the economic value of dry powder inhaler over aerosolised formulation.

Results showed that TIP is associated with lower costs and greater quality-adjusted-life-years as compared with TIS.

Use of TIP over TIS is likely to reduce the economic burden of CF in the USA.

\section{Introduction}

Cystic fibrosis (CF) is a rare, potentially fatal, genetic disorder caused by mutations in the gene encoding the $\mathrm{CF}$ transmembrane conductance regulator protein, which acts as a chloride channel [1]. CF is most common in the Caucasian population, although it can affect nearly every 
race and ethnicity [1]. In the USA, there are over 28,000 paediatric and adult patients with $\mathrm{CF}$ and close to 1000 new cases are diagnosed every year [1]. CF is associated with high socioeconomic costs. Major drivers of the total costs are direct healthcare costs in adults (medications, outpatient healthcare visits, acute hospitalisations) and direct non-healthcare informal costs in children (caregivers' time) $[2,3]$. Healthcare costs increase with the patient's age and severity of the disease [4]. The annual medical cost for a patient with CF in the USA has been reported to be 20 -fold greater than that for someone without CF [5].

Chronic lung infection with Pseudomonas aeruginosa occurs in approximately $50 \%$ of the patients with $\mathrm{CF}$, and is more common in older adolescents and adults [1]. This infection further compromises lung function and significantly contributes to the increased healthcare costs due to more frequent physician visits and extended hospitalisations, as well as non-healthcare informal costs $[1,2,6,7]$. Data show that early diagnosis of $P$. aeruginosa pulmonary infection and aggressive intervention remain key for improving lung function, lowering hospitalisation rates due to acute pulmonary exacerbations, and improving the quality of life (QoL) [8, 9]. The Cystic Fibrosis Pulmonary Guidelines have reported that intravenous antibiotics are the preferred treatment for acute pulmonary exacerbations, while aerosolised or inhaled formulations are preferred for managing chronic pulmonary infections [10].

Tobramycin, an aminoglycoside antibiotic, is used to manage both acute and chronic pulmonary $P$. aeruginosa infections [11]. A recent review, focused primarily on tobramycin inhalation powder (TIP), reinforced that tobramycin inhalation solution (TIS) was efficacious in improving lung function and reducing $P$. aeruginosa sputum density, hospitalisation rates, and the frequency of intravenous antibiotic use [12]. However, its twice-daily administration via a nebuliser for about $15-20 \mathrm{~min} / \mathrm{dose}$, and the additional time required for cleaning and disinfection of the nebuliser is often bothersome and costly [13-15]. This may place a high burden on patients and their caregivers, leading to poor adherence, and possibly reduced effectiveness in real-world settings [16, 17]. Therefore, new treatment options that can reduce the associated patient or caregiver burden are needed.

TIP was the first antibiotic approved by the US Food and Drug Administration (FDA) as a dry powder for inhalation (DPI) formulation for use in patients with CF, and has been recommended as a therapeutic option for the treatment of chronic pulmonary P. aeruginosa infections [18]. Designed to enhance drug delivery, TIP shortens the drug administration time and has been shown to improve patient convenience, satisfaction, and adherence [18, 19]. Although the safety and efficacy profile of TIP was similar to that of TIS over a 6-month period in a randomised, controlled study (EAGER), the administration time for TIP was significantly shorter as compared with TIS (mean 5.6 vs $19.7 \mathrm{~min}$, respectively; $p<0.0001$ ) [15]. TIP was associated with both immediate- and long-term benefits with respect to perceived treatment burden, convenience, adherence, and patient satisfaction, when studied alone or in comparison with TIS $[15,17,20]$.

In a real-world setting, approximately 33 of $40(83 \%)$ patients treated with TIP for 12 months had excellent $(>80 \%)$ adherence and lower intravenous antibiotic usage, a marker used for pulmonary exacerbations, as compared to the previous 12 months [17]. Approximately 94\% of patients who had previously used TIS, preferred TIP over TIS [17]. TIP was also found to have an acceptable longterm safety profile [21].

Considering these findings, it is important to understand the cost-effectiveness of treatments for efficient distribution of healthcare resources. The aim of this analysis was to assess the potential pharmacoeconomic benefit of increased adherence with TIP over TIS in the US setting.

\section{Materials and Methods}

\subsection{Model Structure}

This cost-effectiveness analysis was primarily based on the clinical data from the EAGER study, which was a randomised, open-label, active-controlled, parallel-arm study [15]. Eligible patients were randomised to TIP or TIS in a 3:2 ratio, and treatments were administered for 28 days followed by 28 days off-treatment, for a total period of 6 months [15].

The model was developed from the US healthcare perspective over a time horizon of 10 years, with a cycle length of 6 months. The 10-year time horizon was considered appropriate to account for potential variances in costs and intrinsic health effects, especially given the median predicted survival of CF patients of 39.3 years and the median age of patients in the EAGER study of 24 years $[1,15]$. Other time horizons were also explored (2 and 5 years) to inform shorter term decision making.

The model employed a patient-level simulation rather than a cohort model as the former accounts for patient heterogeneity and allows obtaining unbiased estimates of mean outcomes, thus avoiding the addition of many health states. Moreover, one can reasonably expect a non-linear relationship between patient characteristics (age, baseline lung function, treatment adherence, etc.) and model outcomes [cost and quality-adjusted life-years (QALYs)]. Furthermore, the choice of model structure is consistent with the guidelines published by Brennan et al [22]. 
The parameters considered in this model (Fig. 1) included decline in forced expiratory volume in $1 \mathrm{~s}$ (expressed as $\mathrm{FEV}_{1} \%$ predicted), frequency of pulmonary exacerbations, and overall survival. $\mathrm{FEV}_{1}$ is a reflection of the extent of airway obstruction [23], and the rate of $\mathrm{FEV}_{1}$ decline is a marker of progression of lung impairment in patients with $\mathrm{CF}$, long-term morbidity, and mortality $[24,25]$. The frequency and severity of exacerbations with $\mathrm{FEV}_{1} \%$ predicted $(<40,40-70,>70 \%)$ and treatment adherence were also assessed as these tend to provide a clinically relevant endpoint [24], and are correlated with increased morbidity, compromised QoL, and mortality $[8,26]$. Moreover, pulmonary exacerbations are the major drivers of increased costs $[3,27]$.

This model assumed high adherence to TIP and low adherence to TIS and the adherence categories were defined as low ( $\leq 2$ cycles), medium ( $>2$ to $<4$ cycles), and high ( $\geq 4$ cycles) [16]. This assumption of higher adherence with TIP is based on adherence observed in Harrison et al [17]. The model simulated one patient at random, assigning a full set of risk factors based on the EAGER study population (e.g. age, gender, and baseline $\mathrm{FEV}_{1} \%$ predicted) (Table 1) [15]. The model recorded the costs and health outcomes for each patient for the entire time horizon, and a total of 5000 patients were simulated to ensure stability of the model results. To further enhance the utility and generalisability of this analysis, two additional scenarios were evaluated: high adherence to both TIP and TIS and high adherence to TIP with medium adherence to TIS. For high adherence to TIP and medium adherence to TIS, the rate of

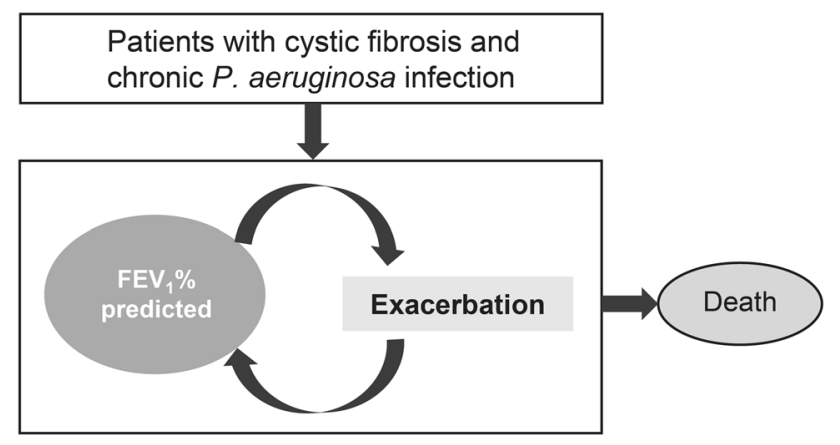

Fig. 1 Model structure. Baseline characteristics used in the model were age, gender, $P$. aeruginosa infection, sputum, crackles, wheezing, sinusitis, elevated liver function tests, and pancreatic insufficiency. Other model inputs included: mean improvement in $\mathrm{FEV}_{1} \%$ predicted $3.1 \%$ for TIP and $2.3 \%$ for TIS; severe exacerbation rate at 6 months with TIP (high adherence): $\mathrm{FEV}_{1} \%<40-1.31, \mathrm{FEV}_{1} \%$ 40-70-0.68, $\mathrm{FEV}_{1} \%>70-0.43$; severe exacerbation rate at 6 months with TIS (low adherence) based on odds ratio of $0.4: \mathrm{FEV}_{1} \%$ $<40-2.04, \mathrm{FEV}_{1} \%$ 40-70-1.23, $\mathrm{FEV}_{1} \%>70-0.85$; mild exacerbation rate at 6 months (considered to be same for TIP and TIS due to lack of data): $\mathrm{FEV}_{1} \%<40-1.32, \mathrm{FEV}_{1} \%$ : 40-70-0.99, $\mathrm{FEV}_{1} \%$ $>70-1.09$. $F E V_{1} \%$ predicted percent predicted forced expiratory volume in $1 \mathrm{~s}$, TIP tobramycin inhalation powder, TIS tobramycin inhalation solution, $P$. aeruginosa Pseudomonas aeruginosa hospitalisation from Briesacher et al [16] was used to calculate the rate of pulmonary exacerbations for TIS based on the rate of exacerbations for high adherence from Bradley et al [28].

The mean (standard deviation) improvement in $\mathrm{FEV}_{1} \%$ predicted was $3.1 \%$ (19.92) for TIP as observed in the EAGER study. Due to the lack of data for improvement of $\mathrm{FEV}_{1} \%$ predicted in patients with low adherence to TIS, the mean improvement in $\mathrm{FEV}_{1} \%$ predicted of $2.3 \%$, which was associated with high adherence to TIS as observed in the EAGER study, was considered as a conservative measure. In addition, the model used the continual longitudinal decline in lung function in $\mathrm{CF}$ patients with chronic $P$. aeruginosa infection. The rate of lung function decline assessed by $\mathrm{FEV}_{1} \%$ predicted was assumed to be constant, and was based on the published studies in patients aged 6-22 years [29, 30], and patients $>18$ years $[31,32]$. The risk factors considered to contribute to lung function decline in children and adolescents in this analysis included crackles, wheezing, sputum production, sinusitis, exacerbations, elevated liver enzymes, and pancreatic insufficiency [32].

Based upon severity, pulmonary exacerbations were classified into two categories, mild (no hospitalisation) and severe (hospitalisation) exacerbations [28]. The rates of severe exacerbations per patient per year based on $\mathrm{FEV}_{1} \%$ predicted $(<40,40-70,>70 \%)$ were obtained from an observational study [28]. These rates were then converted to probabilities of exacerbations $(0,1,2,3$, and 4$)$ per patient in 6 months for TIP. For TIS, due to low adherence, the probabilities of exacerbation per patient were calculated based on the odds ratio of 0.4 (probability of hospitalisations in a patient with high adherence compared to a patient with low adherence) [16]. Similarly, the rates of mild exacerbations per patient per year based on $\mathrm{FEV}_{1} \%$ predicted $(<40,40-70,>70 \%)$ were obtained from the same observational study [28]. These rates were converted to the probability of exacerbations in 6 months for TIP. A conservative assumption was made to consider the same probability of mild exacerbations for TIS (Table 2). The assumption for exacerbation data was based on the observational study [28] rather than the EAGER study. The EAGER study did not measure exacerbations directly; instead, exacerbations were generally reported by the investigators as adverse events using the safety preferred term of lung disorders. Moreover, the EAGER study did not differentiate between minor and major exacerbations.

The mortality rates were estimated using the survival curve reported by Harness-Brumley et al [33], with an appropriate mortality rate assigned to each simulated patient based on their age and gender.

The utility values were derived from the EuroQoL (EQ)$5 \mathrm{D}$ values collected in a UK-based observational study 
Table 1 Baseline patient population characteristics

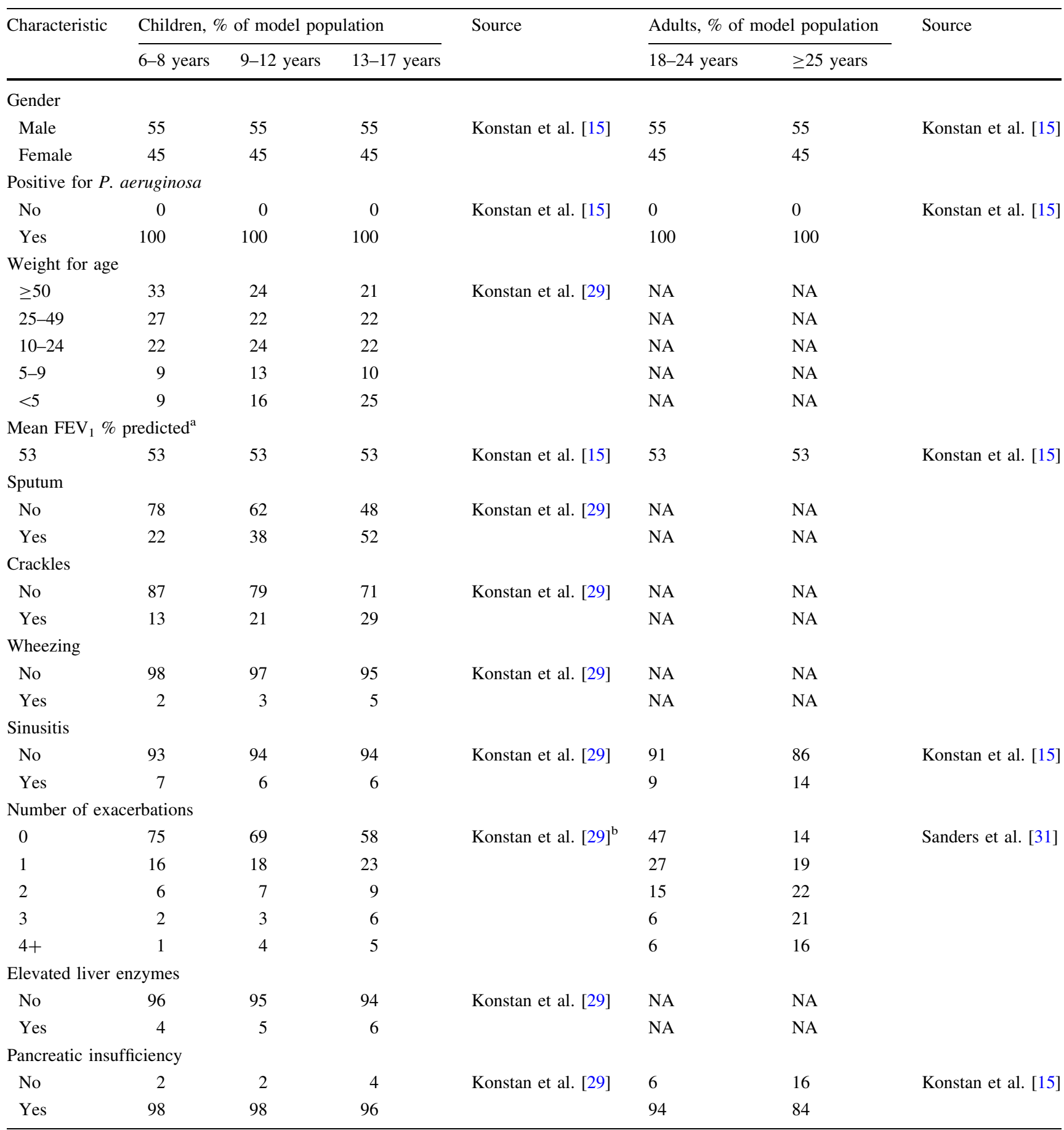

$F E V_{1} \%$ predicted percent predicted forced expiratory volume in $1 \mathrm{~s}, N A$ not applicable for adult population

${ }^{a}$ Mean value for the study cohort, i.e. all patients aged $\geq 6$ years

${ }^{b}$ Baseline distribution of exacerbations is used only in cycle 1

(Table 3) [28]. The EQ-5D data were grouped based on $\mathrm{FEV}_{1} \%$ predicted $(<40,40-70,>70 \%)$ and type of pulmonary exacerbation. Patients in both treatment groups started with the same base utility values, and these would change with the change in $\mathrm{FEV}_{1} \%$ predicted. Disutility (decrement in the normal utility) was applied when an exacerbation occurred. The standard willingness-to-pay (WTP) threshold was considered as US\$50,000-100,000 
Table 2 Mean number of exacerbations by patient, per year

\begin{tabular}{lll}
\hline $\mathrm{FEV}_{1} \%$ predicted severity & Population & \\
\cline { 2 - 3 } level & Requiring hospitalisation (severe) & Treated at home (mild) \\
\hline FEV $_{1} \%$ predicted, $<40$ & 2.61 & 2.64 \\
$\mathrm{FEV}_{1} \%$ predicted, $40-69$ & 1.36 & 1.97 \\
$\mathrm{FEV}_{1} \%$ predicted, $>70$ & 0.86 & 2.18 \\
\hline
\end{tabular}

$F E V_{1} \%$ predicted percent predicted forced expiratory volume in $1 \mathrm{~s}$
Table 3 Utility values for the cystic fibrosis model

\begin{tabular}{lll}
\hline Utility values & & \\
\hline Description & Utility value & Standard deviation \\
\hline FEV $_{1} \%$ predicted, 70-99 & 0.864 & 0.165 \\
FEV $_{1} \%$ predicted, $40-69$ & 0.810 & 0.216 \\
FEV $_{1} \%$ predicted, $<40$ & 0.641 & 0.319 \\
Mild exacerbation $^{\text {a }}$ & 0.060 decrement & 0.048 \\
Severe exacerbation $^{\text {b }}$ & 0.260 decrement & 0.341
\end{tabular}

$F E V_{1} \%$ predicted percent predicted forced expiratory volume in $1 \mathrm{~s}$

${ }^{a}$ Mild exacerbation is defined as any new outpatient antipseudomonal antibiotic without hospitalisation

b Severe exacerbation is defined as respiratory-related hospitalisation

per QALY [34], and a 3\% annual discount rate was applied for both costs and benefits. Clinical experts validated the model structure. After 1 year in the model, characteristics of the patient cohort such as age, gender, risk factors (e.g. sinusitis), and number of exacerbations were similar to those of clinical trial data [15]. Data collection and analysis of the results were also validated using Drummond's checklist [35].

\subsection{Model Assumptions}

The 6-month cycle length was used to match the clinical data in the EAGER study [15]. The treatment administration was considered during the time period the patient was alive or throughout the time horizon, whichever occurred first. The treatment efficacy was considered only in the first cycle due to the lack of long-term data. The safety profile was assumed to be similar for both treatments and was not considered in this model.

\subsection{Resource Use and Costs}

The components included under costs were medication costs, nebuliser costs, regular follow-up costs, and costs of pulmonary exacerbations. The cost-base year was 2016 . The total drug costs for three treatment cycles of TIP and
TIS (Novartis data on file) were taken at US\$26,588 and US $\$ 22,013$, respectively (package price/month US\$8863 and US\$7338, respectively) [36]. Additionally, costs associated with nebuliser use were considered for TIS (Novartis data on file), which included recurrent costs for air compressor and aerosol mask (US\$80, replaced every 6 months), and PARI LC Plus ${ }^{\circledR}$ and air filter (US\$180, replaced every year) [36]. The annual management costs for follow-up were taken at US\$2043 (Table 4). Resource utilisation data for pulmonary exacerbations were obtained from a UK-based study due to the non-availability of similar US data [28]. The resource utilisation parameters comprised the average number of routine and specialist physician visits by provider type and the average number of diagnostic tests, including routine laboratory tests and radiologic investigations [28]. The US-specific unit costs were applied to the resource use data to calculate the costs in US dollar (US\$). The resource use and unit costs are presented in Tables 4 and 5.

\subsection{Model Outputs}

Three categories of model outputs were estimated for each treatment arm: clinical (overall survival, QALYs, and number of pulmonary exacerbations), costs (medication costs, nebuliser costs, regular follow-up costs, exacerbation costs, and total costs), and cost-effectiveness (incremental cost-effectiveness ratio, ICER).

\subsection{Sensitivity Analyses}

As this economic analysis was based on heterogeneous data sources with several built-in assumptions, one-way sensitivity analyses were performed by varying different factors to understand their impact on the outcomes. To arrive at a fair point for eliciting important variables, $95 \%$ confidence interval (CI) was used for the parameters for which a standard deviation was available, and a $10 \%$ standard error was selected for other parameters. A probabilistic sensitivity analysis (PSA) was also performed to further account for uncertainty in the estimated values. The PSA was run for 1000 patients and 100 cohorts using Monte Carlo 
Table 4 Resource use and costs inputs used for the analysis

\begin{tabular}{|c|c|c|c|c|c|}
\hline & $N$ & Mean use ${ }^{b}$ & Unit cost (US\$) & Code & Source \\
\hline \multicolumn{6}{|l|}{ Routine visits in last 12 months } \\
\hline Patients with visits & 90 & & & & \\
\hline If yes, how many? & 66 & 3.3 & 85 & $29-1062$ & Healthcare Salaries [37] \\
\hline \multicolumn{6}{|c|}{ Specialists and frequency of visits in last 12 months (routine outpatient visits) } \\
\hline Specialist 1 -respiratory or $\mathrm{CF}$ & 66 & 2.3 & 200 & & $\begin{array}{l}2009 \text { National Schedule of Reference Cost 2008-09 } \\
\text { NHS Trusts and PCTs combined }\end{array}$ \\
\hline Specialist 2 -diabetes or other & 19 & 1.5 & & & \\
\hline Specialist $3-$ other $^{\mathrm{a}}$ & 9 & 1.4 & & & \\
\hline Specialist 4 -other ${ }^{\mathrm{a}}$ & 4 & 1.3 & & & \\
\hline \multicolumn{6}{|c|}{ Number of visits with other medical staff in last 12 months (routine outpatient visits) } \\
\hline Nurse (CF specialist) & 82 & 1.8 & 31 & $29-1141$ & Bureau of Labor Statistics [38] \\
\hline Physiotherapist & 82 & 2 & 25.82 & $29-1128$ & \\
\hline Dietitian/nutritionist & 81 & 1.2 & 30.5 & $29-1031$ & Bureau of Labor Statistics [38] \\
\hline Psychologist & 87 & 0.1 & 37.07 & $19-3030$ & Bureau of Labor Statistics [38] \\
\hline \multicolumn{6}{|c|}{ Number of routine laboratory tests in last 12 months (during routine visits) } \\
\hline Complete metabolic panel & 90 & 3.2 & 20 & & Clear Health Costs [39] \\
\hline Complete blood count & 90 & 3.2 & & & \\
\hline Pulmonary function tests & 90 & 5.4 & 129 & & Health and Personal Care Costs and Prices Paid [40] \\
\hline Sputum/cough swab cultures & 90 & 3.8 & 67 & & MD Save [41] \\
\hline Other (specify) & 86 & 1.1 & & & \\
\hline \multicolumn{6}{|c|}{ Number of radiology investigations in last 12 months (during routine visits) } \\
\hline Chest X-ray & 90 & 0.7 & 200 & & Health and Personal Care Costs and Prices Paid [42] \\
\hline Other (specify) & 84 & 0.1 & & & \\
\hline Annual management costs & & & 2043 & & \\
\hline
\end{tabular}

NHS National Health Service, PCTs Primary Care Trusts, CF cystic fibrosis

a Other specialist types cited by only one or two patients include: dermatologist, gastroenterologist, gynaecologist, hepatologist, neurogastroenterologist, obstetrician, physiotherapist, psychiatrist, rheumatologist, and surgical specialist

${ }^{\mathrm{b}}$ Count of events and sample size (number of patients) is based on observational study findings (includes number of hospital days per exacerbation, frequency and distribution of antibiotics prescribed, and office encounters)

simulation. Model results were recorded for incremental QALYs gained and incremental cost for TIP versus TIS.

\section{Results}

Patients receiving TIP had better outcomes in terms of number of QALYs and pulmonary exacerbations compared with those receiving TIS, when high adherence to TIP and low adherence to TIS was considered (Fig. 2). For the first additional scenario, where high adherence to both TIP and TIS was assumed, patients receiving TIP had only marginally better outcomes compared with those receiving TIS in terms of the number of exacerbations [mean $(95 \% \mathrm{CI})$; 38.51 (26-53) and 39.45 (30-50), respectively; mean difference -0.94]. However, the overall survival [mean $(95 \%$ CI); 3.40 (0-9.50) years] and QALYs [2.02 (0-5.45)] for both treatments, were similar. For the second additional scenario, where high adherence to TIP and medium adherence to TIS was assumed, patients receiving TIP had better outcomes compared with those receiving TIS in terms of QALYs [mean (95\% CI); $2.10(0-5.52)$ and 1.93 (0-5.12), respectively; mean difference 0.17] and the number of exacerbations [mean (95\% CI); 38.71 (26-54) and 50.63 (40-63), respectively; mean difference -11.92]. Again, the mean $(95 \% \mathrm{CI})$ overall survival was similar for the two treatments [3.54 (0-10.50) years].

The use of TIP over TIS was associated with cost savings per patient over a time horizon of 10 years for nebuliser and exacerbation costs when high adherence to TIP and low adherence to TIS was assumed (Fig. 3). The incremental total costs were lower (by US\$36,168) and QALYs gained were higher (by 0.27 ) with TIP as compared to TIS. This equates to lower costs with TIP per QALY gained (-US\$133,195/QALY) for the base case (ICER). Assuming high adherence to both TIP and TIS, the use of TIP over TIS was associated with marginal cost savings per patient over a time horizon of 10 years for 
Table 5 Resource use and costs of pulmonary exacerbations

\begin{tabular}{|c|c|c|c|c|}
\hline & Events & $\begin{array}{l}\text { No. of } \\
\text { exacerbations }{ }^{a}\end{array}$ & $\begin{array}{l}\text { Cost per unit consumed } \\
\text { (US\$) }\end{array}$ & Source \\
\hline \multicolumn{5}{|l|}{ Severe exacerbations } \\
\hline Hospital days & 1375 & 150 & 1355 & Pasquale et al. $[43]^{\mathrm{b}}$ \\
\hline Admitted through the emergency room & 5 & 150 & 106 & Dewitt et al. [44] \\
\hline Hospital IV antibiotic treatment days & 1563 & 150 & 108 & $\begin{array}{l}\text { Calculated by taking individual price of } \\
\text { antibiotics [45] }\end{array}$ \\
\hline Home IV antibiotics treatment days & 729 & 150 & 177 & $\begin{array}{l}\text { Calculation from individual price of } \\
\text { antibiotics [45] }\end{array}$ \\
\hline Pulmonologist office visits & 3 & 150 & 85 & Healthcare Salaries [37] \\
\hline Nurse specialist & 48 & 150 & 35 & Bureau of Labor Statistics [38] \\
\hline Other follow-up visits & 5 & 150 & & \\
\hline Weighted cost per exacerbation & & & 14,422 & \\
\hline \multicolumn{5}{|l|}{ Mild exacerbations } \\
\hline Home IV antibiotic treatment days & 1643 & 217 & 111 & $\begin{array}{l}\text { Calculated by taking individual price of } \\
\text { antibiotics [45] }\end{array}$ \\
\hline Home oral therapy treatment days & 2176 & 217 & 4 & $\begin{array}{l}\text { Cost of ciprofloxacin } 750 \mathrm{mg} \text { strength } \\
\text { from Analy Source [45] }\end{array}$ \\
\hline Pulmonologist office visits & 5 & 217 & 85 & Healthcare Salaries [37] \\
\hline Nurse specialist & 84 & 217 & 35 & Bureau of Labor Statistics [38] \\
\hline $\begin{array}{l}\text { Other follow-up visits } \\
\text { (including phone calls) }\end{array}$ & 99 & 217 & & \\
\hline Weighted cost per exacerbation & & & 894 & \\
\hline
\end{tabular}

$I V$ intravenous

${ }^{\mathrm{a}}$ Rates of mild and severe exacerbations were obtained from Bradley et al. [28]

${ }^{\mathrm{b}}$ Cost per hospitalisation was calculated based on Table 3 from Pasquale et al, and this cost was converted to 2016 cost based on consumer price index values

Fig. 2 Comparison of health outcomes for treatment with tobramycin inhalation powder and tobramycin inhalation solution. ${ }^{\mathrm{a}}$ Mean difference; ${ }^{\mathrm{b}} 95 \%$ confidence intervals
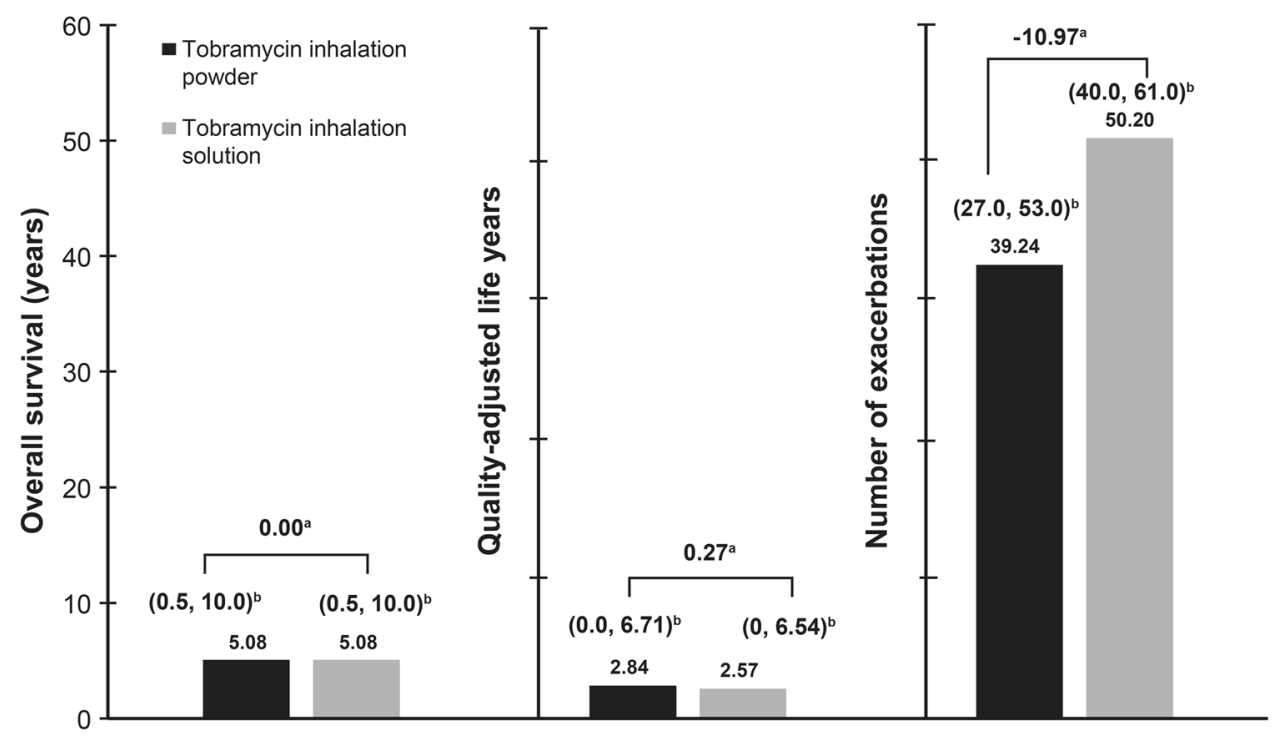

nebuliser and exacerbation costs. The incremental mean total costs $(95 \% \mathrm{CI})$ were higher [by US\$26,217; total costs with TIP and TIS, US $\$ 250,029$ (US\$0-US\$669,893) and US\$223,813 (US\$0-US\$596,081), respectively], but QALYs gained were only marginally higher [by 0.003 ;
QALYs gained with TIP and TIS, $2.024(0-5.47)$ and 2.021 (0-5.46), respectively] with TIP as compared to TIS. This equates to higher costs with TIP per QALY gained (US\$8,555,306/QALY) for the base case (ICER). Assuming high adherence to TIP and medium adherence to TIS, 
use of TIP over TIS was associated with cost savings per patient over a time horizon of 10 years for nebuliser and exacerbation costs. The incremental total costs were lower [by US\$21,865; total costs with TIP and TIS, US\$261,066 (US\$0-US\$687,042) and US\$282,931 (US\$0US\$744,832), respectively] and QALYs gained were higher [by 0.17; QALYs gained with TIP and TIS, 2.10 (0-5.52) and $1.93(0-5.12)$, respectively] with TIP as compared to TIS. This equates to lower costs with TIP per QALY gained (-US\$132,426/QALY) for the base case (ICER).

Compared to TIS, use of TIP increased QALYs by 0.1 and 0.2 years and decreased total costs by US\$11,534 and US\$24,417 at 2 years and 5 years, respectively, with corresponding estimated ICERs of -US\$115,335/QALY and -US\$122,087/QALY.

The one-way sensitivity analyses showed that the probability of severe exacerbation(s) in a patient with high adherence compared to a patient with low adherence (odds ratio) is the most important parameter that could potentially impact the conclusion of this analysis. The lower costs with TIP per QALY gained ranged from -US\$68,554 to US\$174,683 as the odds ratio varied across the CI. Mean improvement in $\mathrm{FEV}_{1} \%$ predicted with TIP serves as the second important parameter followed by utility for patients (QALYs) and rate of severe exacerbations with TIP for patients with $\mathrm{FEV}_{1}$ between 40 and $70 \%$. Across all the scenarios analysed, TIP emerged as a dominant strategy (i.e. more effective and less costly), thereby ensuring the robustness of the current analysis (Fig. 4a). In the PSA results, the incremental QALY values ranged from 0.16 to 0.2 and incremental costs ranged from - US $\$ 20,000$ to
-US\$26,000, and TIP was dominant over TIS in $100 \%$ of the simulations (Fig. 4b).

\section{Discussion}

The results of this pharmacoeconomic assessment showed that TIP is a dominant treatment strategy associated with lower costs and higher QALYs as compared with TIS in CF patients with chronic pulmonary $P$. aeruginosa infection over a 10-year time horizon in the US setting. Similar results were observed over time horizons of 2 and 5 years. These findings were supported by the PSA where across all the simulations run, TIP emerged as the dominant treatment strategy compared to TIS. It is important to note that these cost savings were largely driven by the reduction in pulmonary exacerbation costs with the use of TIP over TIS assuming high adherence to TIP and low adherence to TIS, and partly by costs associated with nebuliser, which is required for administering TIS. These findings were further substantiated by one-way sensitivity analyses considering high adherence to TIP and low adherence to TIS. Published data show that long-term treatment of chronic P. aeruginosa infection in patients with CF is costly, and the overall benefits are highly dependent on the treatment regimen, patient satisfaction, and patient adherence [46]. DPI formulations may have an advantage over the aerosolized formulations in terms of patient satisfaction by shortening the administration time and eliminating the burden of nebuliser maintenance, and therefore, patient adherence [17]. Increased adherence to the treatment may therefore result in better health outcomes such as fewer pulmonary
Fig. 3 Comparison of average costs for treatment with tobramycin inhalation powder (TIP) and tobramycin inhalation solution (TIS).

${ }^{a}$ Confidence intervals. Costs as well as confidence intervals for regular follow-up were the same for the two treatments

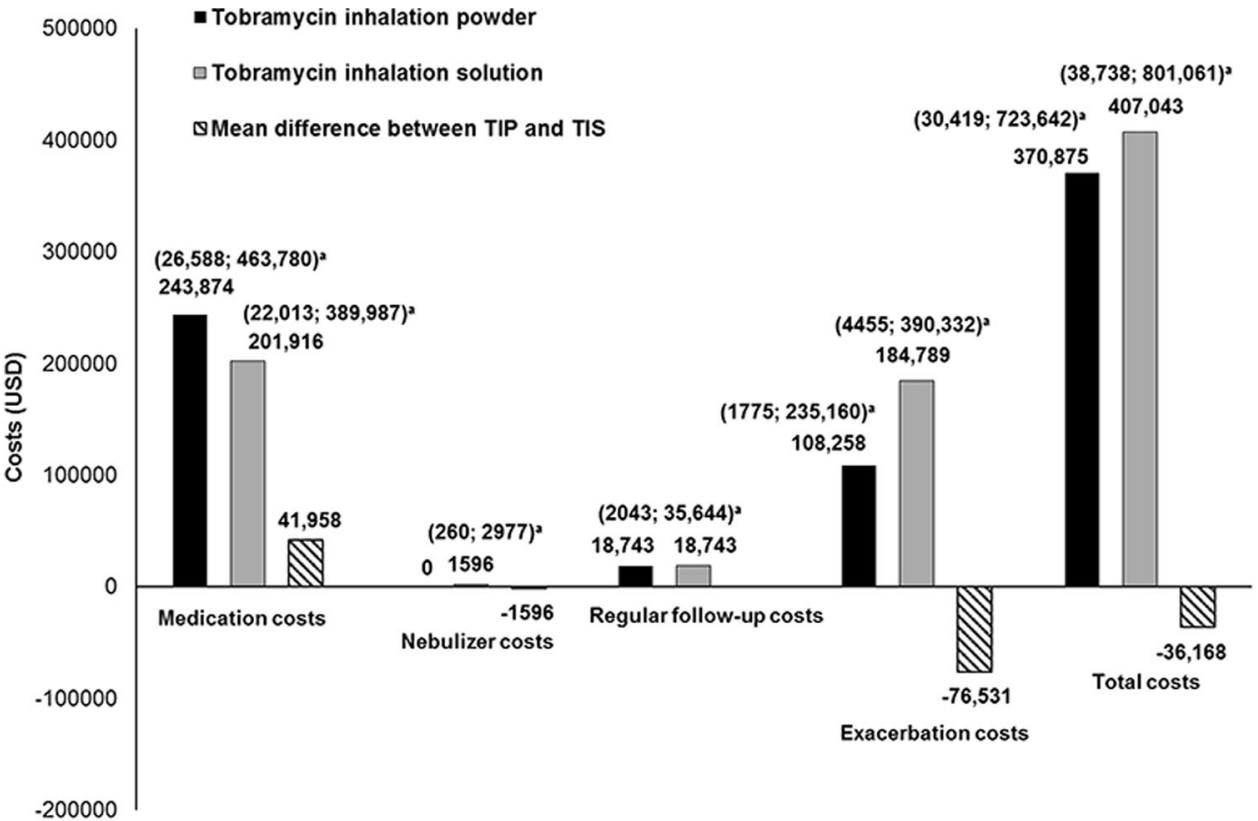


Fig. 4 a One-way sensitivity analyses-tornado chart. b Probabilistic sensitivity analysis. $Q A L Y s$ qualityadjusted life-years, $F E V_{l}$ forced expiratory volume in $1 \mathrm{~s}, T I P$ tobramycin inhalation powder, TIS tobramycin inhalation solution, ICER incremental cost-effectivenes ratio

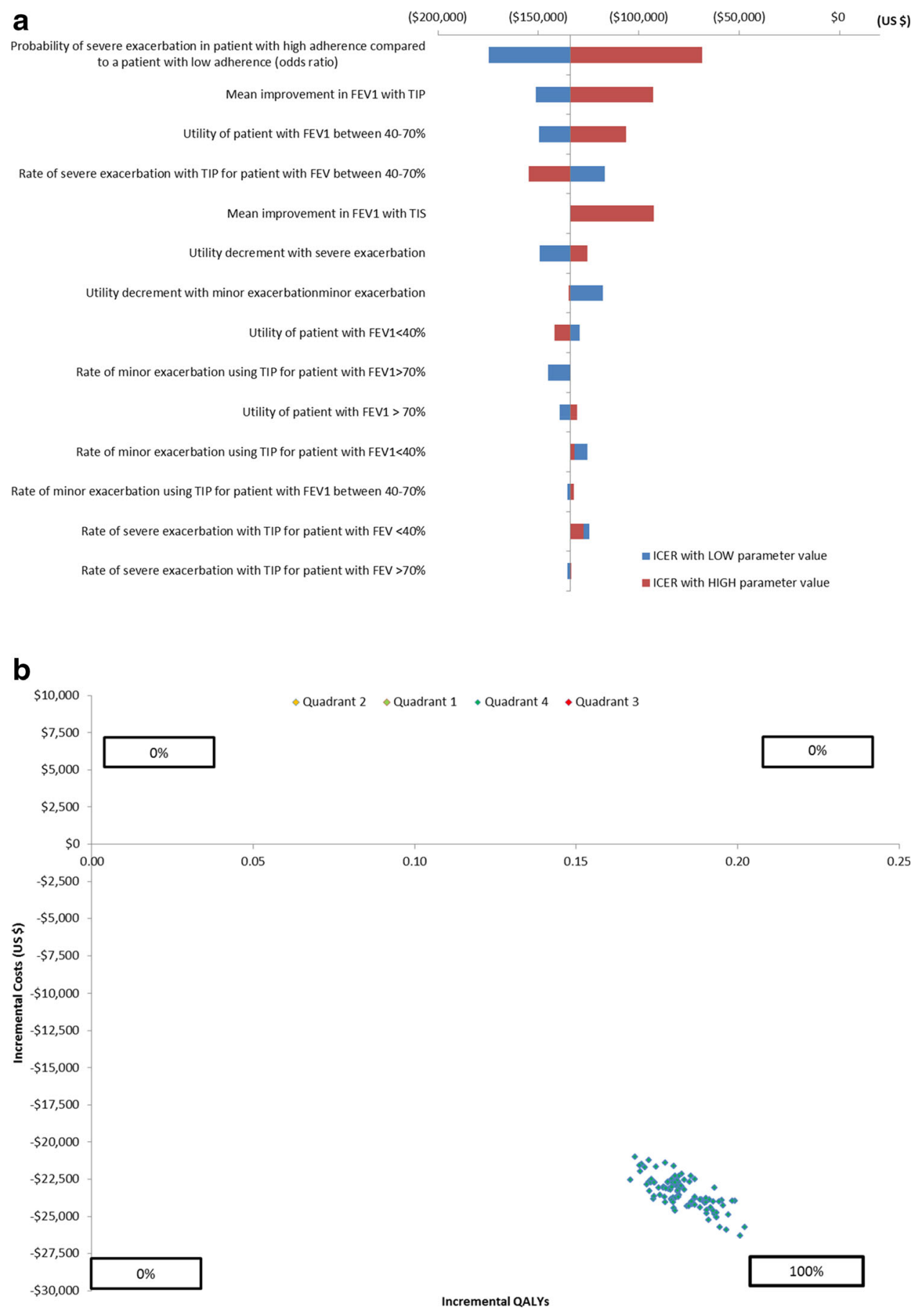

Incremental QALYs exacerbations and hospitalisations. However, there are limited data comparing the clinical and cost-effectiveness outcomes of DPI versus aerosolized formulations in a realworld scenario, thereby limiting the decision making by healthcare practitioners.

The current model was therefore developed to assess the potential health economic benefit of improved adherence with TIP over TIS in the US setting. The model inputs were primarily derived from the EAGER study [15] as this study compared the efficacy of TIP and TIS by evaluating the standard efficacy outcomes, i.e. $\mathrm{FEV}_{1} \%$ predicted in a large population with $\mathrm{CF}$ and chronic pulmonary $P$. aeruginosa infection over a period of 6 months. Another advantage of using the EAGER study for model inputs was that $>60 \%$ of the patients enrolled in the EAGER study were from North America [15], which supports the extrapolation of these data to a larger US population. Inhaled antibiotics other than tobramycin were not used in 
this model as the aim of the analysis was to compare the inhaled versus aerosolized formulation of tobramycin, therefore excluding any potential bias that may arise from evaluating another drug with a different efficacy and safety profile.

The reduction in pulmonary exacerbations, as noted in this analysis, is expected to have a direct impact on QALYs [32]. The QALYs have a high clinical relevance as patients with CF often report a poor HRQoL due to decline in FEV $\%$ predicted and occurrence of pulmonary exacerbations [47]. These analyses suggest that TIP provides increased benefits at lower costs as compared to TIS. However, it should be noted that there are no published data from the USA comparing the cost-effectiveness of DPI and aerosolised solution in patients with CF and chronic pulmonary $P$. aeruginosa infection, precluding any comparisons with published literature. Nevertheless, published studies support the cost-effectiveness and the efficient use of other DPIs in chronic obstructive pulmonary disease [48, 49].

To the best of our knowledge, this is the first cost-effectiveness analysis of TIP versus TIS for treating chronic pulmonary $P$. aeruginosa infection in patients with $\mathrm{CF}$ in the US setting, thus exploring the economic value of DPI over aerosolized formulation. However, the current analysis has several limitations. First, the evaluation was limited by insufficient evidence on resource use and costs in the USA to make adequate comparisons. Resource utilisation and utility data were therefore obtained from a UK-based study, and these were not treatment specific. Second, the model did not assess treatment outcomes over a prolonged period, impact of $P$. aeruginosa sputum density, or longterm treatment adherence. Third, the adherence data obtained from a real-world study conducted shortly after approval of TIP may not reflect current adherence patterns to TIP in the USA $[15,17]$. However, there are no other available data to our knowledge that assessed more recent adherence patterns to TIP.

Nonetheless, the present analysis has a high relevance as the model was based on the best available evidence, and the assumptions were based on published data from real-world studies. Also, the additional analyses performed by varying adherence would help in clinical decision-making according to the different scenarios, with a focus on cost-effectiveness.

\section{Conclusion}

TIP is likely be a more cost-effective treatment strategy than TIS assuming high adherence to TIP, and the use of TIP over TIS may reduce the economic burden of CF. Comprehensive assessment will require long-term comparative data. Nevertheless, the results of this analysis give insight into the cost-effectiveness of TIP for treating chronic pulmonary $P$. aeruginosa infection in patients with CF in the US setting, and hence may allow an efficient distribution of healthcare resources.

Acknowledgements The authors acknowledge Purnima Pathak (Novartis) for providing medical writing support for this manuscript.

Author Contributions All authors were responsible for the development of the cost effectiveness model. In addition, all authors were involved in reviewing and approving the manuscript.

\section{Compliance with Ethical Standards}

Funding The study was funded by Novartis Pharma AG.

Conflict of interest SP and PG are employees of Novartis Healthcare Pvt. Ltd. LD and KH are employees of Novartis Pharmaceuticals Corporation.

Open Access This article is distributed under the terms of the Creative Commons Attribution-NonCommercial 4.0 International License (http://creativecommons.org/licenses/by-nc/4.0/), which permits any noncommercial use, distribution, and reproduction in any medium, provided you give appropriate credit to the original author(s) and the source, provide a link to the Creative Commons license, and indicate if changes were made.

\section{References}

1. Cystic Fibrosis Foundation: https://www.cff.org/2014-AnnualData-Report.pdf (2014). Accessed 17 Aug 2016.

2. Angelis A, Kanavos P, López-Bastida J, Linertová R, Nicod E, Serrano-Aguilar P. Social and economic costs and health-related quality of life in non-institutionalised patients with cystic fibrosis in the United Kingdom. BMC Health Serv Res. 2015;15:428.

3. Chevreul K, Michel M, Brigham KB, López-Bastida J, Linertová R, Oliva-Moreno J, et al. Social/economic costs and health-related quality of life in patients with cystic fibrosis in Europe. Eur J Health Econ. 2016;17(1):7-18.

4. Van Gool K, Norman R, Delatycki MB, Hall J, Massie J. Understanding the costs of care for cystic fibrosis: an analysis by age and health state. Value Health. 2013;16(2):345-55.

5. Ouyang L, Grosse SD, Amendah DD, Schechter MS. Healthcare expenditures for privately insured people with cystic fibrosis. Pediatr Pulmonol. 2009;44(10):989-96.

6. Gu Y, García-Pérez S, Massie J, van Gool K. Cost of care for cystic fibrosis: an investigation of cost determinants using national registry data. Eur J Health Econ. 2015;16(7):709-17.

7. Sansgiry SS, Joish VN, Boklage S, Goyal RK, Chopra P, Sethi S. Economic burden of Pseudomonas aeruginosa infection in patients with cystic fibrosis. J Med Econ. 2012;15(2):219-24.

8. Justicia JL, Solé A, Quintana-Gallego E, Gartner S, de Gracia J, Prados C, et al. Management of pulmonary exacerbations in cystic fibrosis: still an unmet medical need in clinical practice. Expert Rev Respir Med. 2015;9(2):183-94.

9. Mogayzel PJ Jr, Naureckas ET, Robinson KA, Mueller G, Hadjiliadis D, Hoag JB, et al. Cystic fibrosis pulmonary guidelines. Chronic medications for maintenance of lung health. Am J Respir Crit Care Med. 2013;187(7):680-9.

10. Flume PA, Mogayzel PJ Jr, Robinson KA, Goss CH, Rosenblatt RL, Kuhn RJ, et al. Cystic fibrosis pulmonary guidelines: treatment of pulmonary exacerbations. Am J Respir Crit Care Med. 2009;180(9):802-8. 
11. Cheer SM, Waugh J, Noble S. Inhaled tobramycin (TOBI): a review of its use in the management of Pseudomonas aeruginosa infections in patients with cystic fibrosis. Drugs. 2003;63(22):2501-20.

12. Vazquez-Espinosa E, Marcos C, Alonso T, Giron RM, GomezPunter RM, Garcia-Castillo E, et al. Tobramycin inhalation powder (TOBI Podhaler) for the treatment of lung infection in patients with cystic fibrosis. Expert Rev Anti Infect Ther. 2016;14(1):9-17.

13. Geller DE, Konstan MW, Smith J, Noonberg SB, Conrad C. Novel tobramycin inhalation powder in cystic fibrosis subjects: pharmacokinetics and safety. Pediatr Pulmonol. 2007;42(4):307-13.

14. Geller DE, Madge S. Technological and behavioral strategies to reduce treatment burden and improve adherence to inhaled antibiotics in cystic fibrosis. Respir Med. 2011;105(Suppl 2):S24-31.

15. Konstan MW, Flume PA, Kappler M, Chiron R, Higgins M, Brockhaus F, et al. Safety, efficacy and convenience of tobramycin inhalation powder in cystic fibrosis patients: the EAGER trial. J Cyst Fibros. 2011;10(1):54-61. doi:10.1016/j.jcf.2010.10.003.

16. Briesacher BA, Quittner AL, Saiman L, Sacco P, Fouayzi H, Quittell LM. Adherence with tobramycin inhaled solution and health care utilization. BMC Pulm Med. 2011;11:5.

17. Harrison MJ, McCarthy M, Fleming C, Hickey C, Shortt C, Eustace JA, et al. Inhaled versus nebulised tobramycin: a real world comparison in adult cystic fibrosis (CF). J Cyst Fibros. 2014;13(6):692-8.

18. McKeage K. Tobramycin inhalation powder: a review of its use in the treatment of chronic Pseudomonas aeruginosa infection in patients with cystic fibrosis. Drugs. 2013;73(16):1815-27.

19. Vázquez-Espinosa E, Girón RM, Gómez-Punter RM, GarcíaCastillo E, Valenzuela C, Cisneros C, et al. Long-term safety and efficacy of tobramycin in the management of cystic fibrosis. Ther Clin Risk Manag. 2015;11:407-15.

20. Bilton D, Nash EF, Peckham D, Haworth CS, Carroll M, Connett $\mathrm{G}$, et al. An evaluation of treatment burden following initiation of TOBI $^{\circledR}$ Podhaler $^{\circledR}$ in patients with CF. J Cyst Fibros. 2014;13(2):S65.

21. Sommerwerck U, Virella-Lowell I, Angyalosi G, Viegas A, Cao W, Debonnett L. Long-term safety of tobramycin inhalation powder in patients with cystic fibrosis: phase IV (ETOILES) study. Curr Med Res Opin. 2016;32(11):1789-95.

22. Brennan A, Chick SE, Davies R. A taxonomy of model structures for economic evaluation of health technologies. Health Econ. 2006;15(12):1295-310.

23. FDA Guidance. 2016. http://www.fda.gov/downloads/drugs/ guidancecomplianceregulatoryinformation/guidances/ucm071575. pdf. Accessed 17 Aug 2016.

24. EMA Guidance. 2009. http://www.ema.europa.eu/docs/en_GB/ document_library/Scientific_guideline/2009/12/WC500017055. pdf. Accessed 17 Aug 2016.

25. FDA Case Study. 2013. http://static1.1.sqspcdn.com/static/f/ 222669/23140976/1374205883667/FDAMissingData2.pdf?token= m1nZf1LVXjTQIsPWK5jV0aROxr4\%3D. Accessed 17 Aug 2016.

26. Solem CT, Vera-Llonch M, Liu S, Botteman M, Castiglione B. Impact of pulmonary exacerbations and lung function on generic health-related quality of life in patients with cystic fibrosis. Health Qual Life Outcomes. 2016;14:63.

27. Bhatt JM. Treatment of pulmonary exacerbations in cystic fibrosis. Eur Respir Rev. 2013;22(129):205-16.

28. Bradley JM, Blume SW, Balp MM, Honeybourne D, Elborn JS. Quality of life and healthcare utilisation in cystic fibrosis: a multicentre study. Eur Respir J. 2013;41(3):571-7.

29. Konstan MW, Morgan WJ, Butler SM, Pasta DJ, Craib ML, Silva SJ, et al. Risk factors for rate of decline in forced expiratory volume in one second in children and adolescents with cystic fibrosis. J Pediatr. 2007;151(2):134-9.

30. Que C, Cullinan P, Geddes D. Improving rate of decline of FEV1 in young adults with cystic fibrosis. Thorax. 2006;61(2):155-7.

31. Sanders DB, Bittner RC, Rosenfeld M, Redding GJ, Goss CH. Pulmonary exacerbations are associated with subsequent FEV1 decline in both adults and children with cystic fibrosis. Pediatr Pulmonol. 2011;46(4):393-400.

32. Konstan MW, Wagener JS, Vandevanter DR, Pasta DJ, Yegin A, Rasouliyan L, et al. Risk factors for rate of decline in FEV1 in adults with cystic fibrosis. J Cyst Fibros. 2012;11(5):405-11.

33. Harness-Brumley CL, Elliott AC, Rosenbluth DB, Raghavan D, Jain R. Gender differences in outcomes of patients with cystic fibrosis. J Womens Health (Larchmt). 2014;23(12):1012-20.

34. Shiroiwa T, Sung YK, Fukuda T, Lang HC, Bae SC, Tsutani K. International survey on willingness-to-pay (WTP) for one additional QALY gained: what is the threshold of cost effectiveness? Health Econ. 2010;19(4):422-37.

35. Drummonds checklist. 1996. http://bmjopen.bmj.com/content/ suppl/2011/12/21/bmjopen-2011-000363.DC1/Research_checklist. pdf. Accessed 17 Aug 2016.

36. FDB MedKnowledge, updated June 2016. https://www. analysource.com/. Accessed with permissions and copyrighted by First Databank, Inc. Accessed 17 Aug 2016.

37. Healthcare Statistics. http://www.healthcare-salaries.com/ physicians/pulmonologist-salary. Accessed 17 Aug 2016.

38. Bureau of Labor Statistics. http://www.bls.gov/. Accessed 17 Aug 2016.

39. Clear Health Costs. http://clearhealthcosts.com/search/?query= $\mathrm{CBC}+$ blood + test $\&$ state $=\&$ rzip - distance $=100 \&$ rzip $=10036 \& \mathrm{x}=$ $0 \& y=0 \&$ latitude $=\&$ longitude $=$. Accessed 17 Aug 2016.

40. Health and Personal Care Costs and Prices Paid. http://health. costhelper.com/pulmonary-function-tests.html. Accessed 17 Aug 2016.

41. MDsave. https://www.mdsave.com/procedures/sputum-culture1/ d483fccc. Accessed 17 Aug 2016.

42. Health and Personal Care Costs and Prices Paid. http://health. costhelper.com/chest-x-rays.html. Accessed 17 Aug 2016.

43. Pasquale MK, Sun SX, Song F, Hartnett HJ, Stemkowski SA. Impact of exacerbations on health care cost and resource utilization in chronic obstructive pulmonary disease patients with chronic bronchitis from a predominantly Medicare population. Int J Chron Obstruct Pulmon Dis. 2012;7:757-64.

44. Dewitt EM, Grussemeyer CA, Friedman JY, Dinan MA, Lin L, Schulman KA, et al. Resource use, costs, and utility estimates for patients with cystic fibrosis with mild impairment in lung function: analysis of data collected alongside a 48 -week multicenter clinical trial. Value Health. 2012;15(2):277-83.

45. Analy Source. https://www.analysource.com/. Accessed 17 Aug 2016.

46. Eakin MN, Bilderback A, Boyle MP, Mogayzel PJ, Riekert KA. Longitudinal association between medication adherence and lung health in people with cystic fibrosis. J Cyst Fibros. 2011;10(4):258-64

47. Dill EJ, Dawson R, Sellers DE, Robinson WM, Sawicki GS. Longitudinal trends in health-related quality of life in adults with cystic fibrosis. Chest. 2013;144(3):981-9.

48. Campbell CT, Hoffman JM, Carlson AA, Besignano AR, Lento GM, Edwards RA. Cost-effectiveness of aclidinium bromide compared with tiotropium bromide from the perspective of COPD patient populations. Int $\mathrm{J}$ Med Pharmacy. 2014;2(2):11-36.

49. Briggs AH, Glick HA, Lozano-Ortega G, et al. Is treatment with ICS and LABA cost-effective for COPD? Multinational economic analysis of the TORCH study. Eur Respir J. 2010;35(3):532-9. 